\title{
Música tradicional en el aula: aportaciones para un aprendizaje significativo en la
} escuela

Traditional music in the classroom: contributions for meaningful learning at school

\author{
Hita Ximena Valverde Ocariz \\ ximena.valverde@gmail.com \\ Departamento de Didáctica de la Expresión Musical, Plástica y Corporal \\ Universidad Autónoma de Barcelona \\ Barcelona, España \\ ORCID: https://orcid.org/0000-0001-9197-8044 \\ Pere Godall \\ Departamento de Didáctica de la Expresión Musical, Plástica y Corporal \\ Universidad Autónoma de Barcelona \\ Barcelona, España \\ ORCID: http://orcid.org/0000-0002-6548-2624
}

doi: 10.7203/LEEME.41.10530

Recibido: 27-11-17 Aceptado: 08-03-18. Contacto y correspondencia: Hita Ximena Valverde Ocariz, Departamento de Didáctica de la Expresión Musical, Plástica y Corporal Universidad Autónoma de Barcelona, Campus de la UAB, Plaça Cívica, 08193 Bellaterra, Barcelona. España.

\section{Resumen}

La educación musical en Chile necesita tener como referentes para el aprendizaje el rico patrimonio de la música tradicional. Este artículo tiene como objetivo analizar la utilización de músicas tradicionales en el nivel de sexto año básico de escuelas chilenas. El diseño metodológico se basa, por una parte, en el análisis de los programas de estudio elaborados por el Ministerio de Educación de Chile y, por la otra, en una encuesta realizada a una muestra de 113 profesores que realizan la clase de música en el nivel de sexto básico, que pertenecen a 13 de las 15 regiones chilenas. En los resultados, se categoriza al profesorado en función de su posicionamiento en la enseñanza de estas músicas, se describe el rol del Estado como educador y transmisor de cultura en la escuela y los datos muestran el interés por el trabajo con músicas tradicionales en el aula.

Palabras clave: Música tradicional, educación musical, interdisciplinariedad

\section{Abstract}

Music education in Chile also needs to have as reference for learning the rich heritage of traditional music. This article aims at the presence and use of traditional music at the sixth grade basic level of Chilean schools. The methodological design is based, on the one hand, on the analysis of the curricula elaborated by the Chilean Ministry of Education and, on the other hand, on a survey carried out on a sample of 113 sixth-grade teachers belonging to 13 of the 15 Chilean regions. In the results, teachers are categorized according to their position in the teaching of these types of music, the role of the State as educator and transmitter of culture in the school is described, and the data is revealed that demonstrates interest in working with traditional music in the classroom

Key words: Traditional music, music education, Interdisciplinarity.

(c) HITA XIMENA VALVERDE OCARIZ Y PERE GODALL. THE CONTENT OF THIS ARTICLE IS THE SOLE RESPONSIBILITY OF THE AUTHORS. THE REVISTA ELECTRÓNICA DE LEEME AND UNIVERSITAT DE VALĖNCIA ARE NOT LIABLE FOR ANY LEGAL ACTIONS THAT MAY ARISE INVOLVING THE ARTICLE'S CONTENT. REVISTA ELECTRÓNICA DE LEEME -LISTA ELECTRÓNICA EUROPEA DE MÚSICA EN LA EDUCACIÓN-HTTP://MUSICA.REDIRIS.ES.ISSN: 1575-9563 EDITORES: UNIVERSIDAD DE VALENCIA Y JESÚS TEJADA GIMÉNEZ. VISIBILIDAD DE ESTA MIAR, OCLC WORLDCAT, RESH, REDIB, RILM CORE JOURNALS, SUDOC, ULRICHS, ESTA REVISTA ESTÁ PUBLICADA CON EL APOYO INSTITUCIONAL DE REDIRIS-CONSEJO SUPERIOR DE INVESTIGACIONES CIENTIFICAS Y ES DE ACCESO LIBRE. CREATIVE COMMONS LICENSE 4.0 BY 


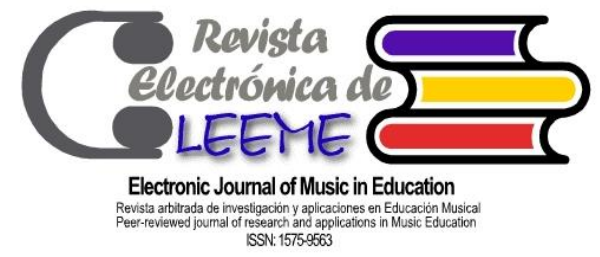

HITA XIMENA VALVERDE OCARIZ Y PERE GODALL

\section{Introducción}

La educación musical dentro del currículum formal chileno ha ido perdiendo espacios y, lamentablemente, se ha vuelto cada vez más prescindible. Un ejemplo de ello quedó demostrado en el año 2011 cuando se promulgó el Decreto Presidencial 1363, que realizó cambios como la cantidad de horas de educación musical. En segundo ciclo de Enseñanza Básica (incluidas las horas para sexto básico), las clases quedaron conformadas por 45 minutos semanales. Por su parte, el Ministerio de Educación (MINEDUC), en 2012, presentó los nuevos programas de estudio para la educación musical desde primero a sexto año básico incluyendo cambios de forma y fondo como, por ejemplo, la no inclusión de unidades de trabajo temáticas, entre ellas, la música tradicional.

El presente artículo se basa en una investigación que pretende analizar la utilización de músicas tradicionales en el nivel de sexto año básico en escuelas chilenas. Para ello, la recolección de información se ha realizado, por un lado, a partir del análisis de programas de educación musical desarrollados por el Ministerio de educación de Chile y, por el otro, de los datos obtenidos con una encuesta realizada a 113 profesores que imparten la asignatura de música en el nivel de sexto año básico en Chile.

\section{La música tradicional como recurso para la educación musical}

Entre las distintas definiciones de lo que se considera como música tradicional, destacamos la de Salazar (2016) quien concreta que son aquellas músicas que forman parte de la cultura e identidad de un grupo social. Por este motivo, siguiendo lo propuesto por Martí (2000) al referirse a músicas tradicionales o folclóricas en el aula, estas deben involucrar no tan solo el repertorio sino, además, un acercamiento a la cultura donde se desarrolla la música estudiada. La música tradicional, tiene su referencia principal en el concepto de "tradición", una idea de tradición desarrollada especialmente durante el romanticismo. Se refiere a un concepto complejo para ser definido y que en ocasiones hace que se entienda la idea de "música tradicional" de una manera tautológica (Martí, 2000).

La valoración de la utilización de músicas tradicionales en la escuela es una necesidad que ha surgido por parte de la comunidad académica internacional desde hace ya décadas (Campbell, 2013). Al respecto, es importante mencionar la valoración que ha tenido a lo largo de los años la relación interdisciplinar que ha ido realizándose entre la educación musical y la etnomusicología (Campbell, 2013; Salazar, 2016). Ha sido desde esta disciplina donde ha nacido el interés de llevar estas músicas al ámbito educacional con el objetivo de conocer la cultura y los contextos en que se crean y desarrollan las músicas en el mundo.

(c) HITA XIMENA VALVERDE OCARIZ Y PERE GODALL. THE CONTENT OF THIS ARTICLE IS THE SOLE RESPONSIBILITY OF THE AUTHORS. THE REVISTA ELECTRÓNICA DE LEEME AND UNIVERSITAT DE VALĖNCIA ARE NOT LIABLE FOR ANY LEGAL ACTIONS THAT MAY ARISE INVOLVING THE ARTICLE'S CONTENT. REVISTA ELECTRÓNICA DE LEEME -LISTA ELECTRÓNICA EUROPEA DE MÚSICA EN LA EDUCACIÓN-HTTP://MUSICA.REDIRIS.ES.ISSN: 1575-9563 EDITORES: UNIVERSIDAD DE VALENCIA Y JESÚS TEJADA GIMÉNEZ. VISIBILIDAD DE ESTA MIAR, OCLC WORLDCAT, RESH, REDIB, RILM CORE JOURNALS, SUDOC, ULRICHS, ESTA REVISTA ESTA PUBLICADA CON EL APOYO INSTITUCIONAL DE REDIRIS-CONSEJO SUPERIOR DE INVESTIGACIONES CIENTIFICAS Y ES DE ACCESO LIBRE. CREATIVE COMMONS LICENSE 4.O BY 


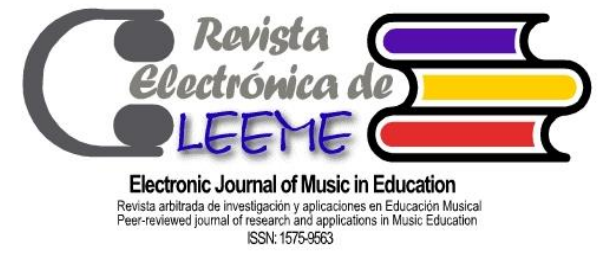

HITA XIMENA VALVERDE OCARIZ Y PERE GODALL

La presencia y utilización de recursos provenientes de las músicas tradicionales en la escuela puede plantear un modelo de transmisión cultural que esté directamente relacionado con la conservación de los saberes y esté basado en la interiorización de las habilidades y los conocimientos de la cultura (Davis, 2009). Serán los hechos musicales quienes participen de manera significativa en la construcción de las identidades por el hecho de ser una expresión viva de un colectivo social. De esta forma se puede llegar a comprender, desde la dimensión educativa, la función de desarrollo identitario y el valor de contribuir desde la sonoridad a la integración de las prácticas sociales de la comunidad a la que pertenecen (Heargraves, 1998). Por su parte, Casals (2009) plantea que la importancia de la música tradicional como un aprendizaje escolar debe basarse en conocer y respetar el propio entorno y la propia cultura, así como para incorporar elementos de otras culturas.

A nivel curricular la experiencia de un aprendizaje a partir de un contacto intercultural o multicultural permite el estudio y la comprensión de conceptos fundamentales de la música y la cultura, ya que la música participa dialógicamente en la creación y renovación de esta (Anderson y Campbell, 1996; Lines, 2009). Las particularidades de las músicas tradicionales permitirán a los estudiantes vivir la cultura musical y participar a partir de la experiencia de la música. Esta experiencia musical dependerá de un concepto generado social o históricamente de que los patrones sonoros deben contar como música (Green, 2008: 32). Es importante que esta experiencia de aprendizaje tenga un carácter global, dado que en muchas músicas tradicionales la danza y la música se mezclan y forman una unidad inseparable. Esto a nivel educacional y de búsqueda de aprendizajes se traduce en una estrecha relación entre la actividad motora y la actividad mental, lo cual posiblemente facilite y mejore el aprendizaje conceptual (Anderson y Campbell, 1996: 7).

\section{La música tradicional en el sistema educativo chileno}

En el contexto educacional chileno, la música tradicional ha estado presente en los programas de estudio elaborados por el Ministerio de Educación con una presencia explícita desde la década de 1930 (Pino, 2015). Sin embargo, a lo largo de la historia de la educación musical en el país, esta presencia ha ido variando según los contextos sociales que se han ido desarrollando en el país. Por ejemplo, durante el periodo comprendido entre 1973 y 1989, donde Chile fue gobernado por una dictadura militar, la mayor parte de las músicas de raíz folclórica fueron eliminadas del currículum escolar y también de toda práctica social pública, por considerarlas subversivas o de incitación al marxismo, con el claro objetivo de contribuir a la despolitización de la sociedad chilena (Donoso, 2009; Poblete, 2016). Esta fue una reacción a lo que sucedió durante el gobierno que precedió a la dictadura, donde se dio un gran énfasis a las músicas de raíz folclórica tanto de Chile como de otros lugares de Latinoamérica (Donoso, 2009). Las músicas tradicionales tendrían a partir de este momento un nuevo lugar, el cual tenía un carácter más decorativo y estilizado. A nivel educativo esto sería especialmente utilizado para reforzar elementos patrios y de identidad nacional (Donoso, 20090; Pino 2015).

(c) HITA XIMENA VALVERDE OCARIZ Y PERE GODALL. THE CONTENT OF THIS ARTICLE IS THE SOLE RESPONSIBILITY OF THE AUTHORS. THE REVISTA ELECTRÓNICA DE LEEME AND UNIVERSITAT DE VALĖNCIA ARE NOT LIABLE FOR ANY LEGAL ACTIONS THAT MAY ARISE INVOLVING THE ARTICLE'S CONTENT. REVISTA ELECTRÓNICA DE LEEME -LISTA ELECTRÓNICA EUROPEA DE MÚSICA EN LA EDUCACIÓN-HTTP://MUSICA.REDIRIS.ES.ISSN: 1575-9563 EDITORES: UNIVERSIDAD DE VALENCIA Y JESÚS TEJADA GIMÉNEZ. VISIBILIDAD DE ESTA MIAR, OCLC WORLDCAT, RESH, RITEFACTOR, COPAC, DIALNET, DICE (CSIC), DOAJ, E-REVISTAS (CSIC), EUSLOADA CON EL APOYO INSTITUCIONAL DE REDIRIS-CONSEJO SUPERIOR DE INVESTIGACIONES CIENTIFICAS Y ES DE ACCESO LIBRE. CREATIVE COMMONS LICENSE 4.0 BY 


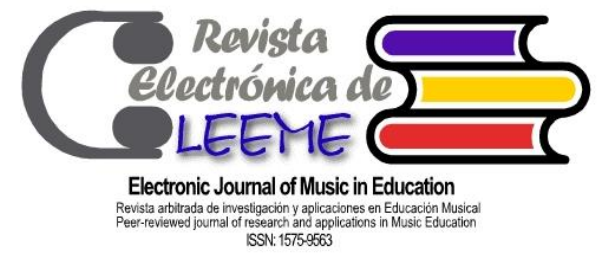

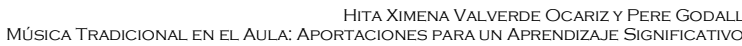
(REVISTA ELECTRÓNICA DE LEEME) NÚMERO 41 , PP. $16-34$ HTTPS://OJS.UV.ES/INDEX.PHP/LEEME/INDEX

Es importante mencionar que, durante la década posterior a la dictadura, se realizaron importantes cambios curriculares, los cuales se vieron reflejados en la elaboración de nuevos programas de estudio para la educación musical del año 2000 donde, por primera vez, se abordan en profundidad temáticas en torno a las músicas tradicionales tanto de pueblos originarios de Chile como también de otros puntos de Latinoamérica. Sin embargo, en el año 2012, nuevamente, los programas de estudio fueron modificados, desapareciendo de esta forma el tratamiento en profundidad que se le otorgaba a la música tradicional.

Por otro lado, el hecho que se pueda contar con programas de estudio para la clase de música, donde se aborde con mayor o menor profundidad la música tradicional, no significa una garantía de que estas músicas lleguen a los estudiantes, ya que aún se debe sortear un obstáculo: la formación previa que han tenido los profesores que realizan las clases de música. Estos debido a que en Chile no existe un currículum nacional para las carreras universitarias (Insunza, Assael y Scherping, 2011). La consecuencia de esta decisión se hace visible al momento del egreso de los profesores desde las universidades ya que cuentan con perfiles profesionales variados y llegan a las aulas con conocimientos muy diferentes.

Es posible encontrar en los sitios web de las universidades chilenas donde se imparte la carrera de Pedagogía en Música que, por ejemplo, en tres ellas no existe ninguna asignatura relacionada con la música tradicional. Por otro lado, universidades tales como la Universidad de Playa Ancha (Valparaíso), Universidad Academia de Humanismo Cristiano (Santiago) o Universidad Inacap (Santiago), destacan porque son las que cuentan con mayor cantidad de asignaturas y horas destinadas a la formación docente en el área de músicas tradicionales.

Es importante destacar que en Chile existen algunos proyectos educativos donde se busca de manera clara y concisa la valoración e inclusión de estas músicas en el aprendizaje escolar. Destacamos dos ejemplos correspondientes a dos lugares de Chile (Valverde, 2013). El primero lo podemos encontrar en el colegio artístico "Santa Cecilia" de la ciudad de Osorno, en el P.E.I. elaborado por un equipo de docentes hace ya más de una década. La enseñanza transversal de músicas latinoamericanas folclóricas y tradicionales se transformó en la columna vertebral de su enseñanza general. Por otra parte, en el norte de Chile, en Iquique, existe el Liceo Artístico "Violeta Parra" cuyo eje transversal de los aprendizajes se nutre de la cultura tradicional. En este caso específico ya no solamente se trata de aprendizaje de músicas, sino que, igualmente, abarca un espectro sociocultural mucho más amplio.

En lo referido a los materiales que actualmente disponen los docentes de música para incluir la música tradicional en las clases estos se encuentran principalmente en sitios web y se pueden consultar y extraer de forma gratuita, desde portales como educarchile, folklore, musicadechile o memoriachilena entre otros; todos ellos ofrecen una variedad importante de recursos y herramientas para el trabajo en el aula. En el sitio web del Ministerio de Educación, no existen canales directos de apoyo con materiales sobre dichas temáticas, si se incluye una

(c) HITA XIMENA VALVERDE OCARIZ Y PERE GODALL. THE CONTENT OF THIS ARTICLE IS THE SOLE RESPONSIBILITY OF THE AUTHORS. THE REVISTA ELECTRÓNICA DE LEEME AND UNIVERSITAT DE VALĖNCIA ARE NOT LIABLE FOR ANY LEGAL ACTIONS THAT MAY ARISE INVOLVING THE ARTICLE'S CONTENT. REVISTA ELECTRÓNICA DE LEEME -LISTA ELECTRÓNICA EUROPEA DE MÚSICA EN LA EDUCACIÓN-HTTP://MUSICA.REDIRIS.ES.ISSN: 1575-9563 EDITORES: UNIVERSIDAD DE VALENCIA Y JESÚS TEJADA GIMÉNEZ. VISIBILIDAD DE ESTA REVISTA: EBSCO, CINDOC (CESIC), CITEFACTOR, COPAC, DIALNET, DICE (CSIC), DOAJ, E-REVISTAS (CSIC), EBSCO PREMIER, ERIH+, GALE CENGAGE LEARNING, IN-RECS, IRESIE, LATINDEX, MIAR, OCLC WORLDCAT, RESH, REDIB, RILM CORE JOURNALS, SUDOC, ULRICHS, ESTA REVISTA ESTÁ PUBLICADA CON EL APOYO INSTITUCIONAL DE REDIRIS-CONSEJO SUPERIOR DE INVESTIGACIONES CIENTIFICAS Y ES DE ACCESO LIBRE. CREATIVE COMMONS LICENSE 4.0 BY 


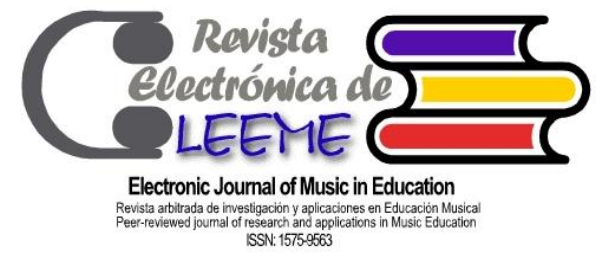

HITA XIMENA VALVERDE OCARIZ Y PERE GODALL

selección de repertorio y propuestas de actividades que existen en los propios programas de estudio. Y, en lo que se refiere a material bibliográfico de apoyo como libros de música tradicional para trabajar en el aula, podemos encontrar algunos materiales como los del profesor Jorge Rodríguez Gallardo, titulado Cantando a Chile y El folclor musical en la escuela, o el material de repertorio llamado Latinoamérica en el aula del equipo de investigación Entorno Sonoro, entre otros. Pese a estas iniciativas, este tipo de material específico sobre músicas tradicionales de Chile o Latinoamérica, hoy por hoy, es aún muy escaso.

Resulta igualmente importante mencionar algunos ejemplos concretos de la aplicación didáctica de músicas tradicionales en la escuela, llevadas a cabo en diferentes puntos de España, donde se hace manifiesta la intención por parte de organismos institucionales de que la música tradicional esté presente y sea parte de los aprendizajes escolares, es el caso de la inclusión de la música tradicional canaria y todos sus elementos interculturales (Delgado, 2005) o la utilización de cantos tradicionales en Jaén (Arévalo, 2009), entre otros.

\section{Método}

La investigación se estructuró a partir de dos fases de trabajo. La primera fase consistió en el análisis de documentos realizado a partir de los programas de estudio de música para sexto año básico del año 2000 y del año 2012: se seleccionó el nivel de sexto básico debido a que, por más de una década, fue el nivel de enseñanza donde se abordaba y otorgaba con mayor profundidad aprendizajes en torno a las músicas tradicionales. Y, la segunda fase, y a partir de los datos obtenidos en el análisis documental, consistió en la elaboración de un cuestionario el cual fue aplicado a profesores que realizaban la clase de música en sexto año básico.

Este cuestionario contó con un total de 28 preguntas, organizadas en 6 partes (identificación de participante, conocimiento del programa de estudio, presencia de músicas tradicionales en $6^{\circ}$ básico, estrategias utilizadas por el docente para el tratamiento de estas músicas, interés del docente por difundir estas músicas, preguntas de cierre y evaluación del instrumento). El cuestionario fue previamente validado por 3 expertos en educación de Chile y aplicado posteriormente a modo de prueba piloto a 3 profesores (anexo 1). En este artículo han sido consideradas 5 preguntas del cuestionario $(15,18,22,23$ y 28$)$, debido a que son las que se relacionan con el tema que se analiza en el estudio: la utilización de estas músicas en el nivel de sexto básico.

\section{Fase 1: Análisis de los programas de estudio}

El análisis consistió, por una parte, en reconocer e identificar los diferentes componentes con que contaba el material recogido y, en un paso posterior, se efectuaron comparaciones entre 


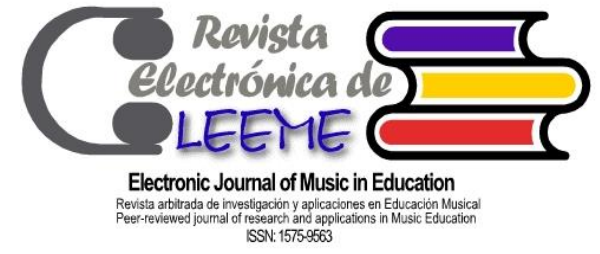

el actual y el anterior programa de estudio para el mismo nivel. Del mismo modo, se generaron categorías para su posterior clasificación y comprensión. A continuación, se presenta un cuadro resumen con los pasos utilizados para el análisis de los programas de estudio.

Tabla 1. Resumen del análisis de programas de estudio

\begin{tabular}{|l|l|}
\hline \multicolumn{1}{|c|}{ Qué se busca identificar } & \multicolumn{1}{c|}{ Qué procedimiento se utiliza } \\
\hline $\begin{array}{l}\text { Principales características de ambos } \\
\text { programas. }\end{array}$ & $\begin{array}{l}\text { Se analizó la presentación del material en ambos programas de estudio } \\
\text { por parte del Ministerio de Educación. }\end{array}$ \\
\hline $\begin{array}{l}\text { Principales diferencias entre ambos } \\
\text { programas }\end{array}$ & $\begin{array}{l}\text { Se compararon los contenidos presentes en ambos programas y cómo } \\
\text { éstos influyen en la importancia y desarrollo de determinados tópicos } \\
\text { y la disminución y/o ausencia de otros. }\end{array}$ \\
\hline Tratamiento de conceptos sobre música & $\begin{array}{l}\text { Se compararon diferentes conceptos musicales y cómo son abordados } \\
\text { en ambos programas de estudio. }\end{array}$ \\
\hline Tratamiento de la música tradicional & $\begin{array}{l}\text { Se comparó la organización y planificación de ambos programas en } \\
\text { relación a la música tradicional: la distribución temporal, la } \\
\text { organización por unidades de aprendizaje, los objetivos y el nivel de } \\
\text { profundidad en los dos programas. }\end{array}$ \\
\hline $\begin{array}{l}\text { Recursos que se utilizan para el tratamiento } \\
\text { de las músicas tradicionales en ambos } \\
\text { programas de estudio. }\end{array}$ & $\begin{array}{l}\text { Se analizaron los recursos propuestos en ambos programas y cómo } \\
\text { éstos contribuyen a la profundización y tratamiento de la música } \\
\text { tradicional en el aula de música. }\end{array}$ \\
\hline
\end{tabular}

\section{Fase 2: Cuestionario para docentes}

Una vez analizado el programa, se extrajeron de él las ideas conductoras para la elaboración de un cuestionario que fue respondido por 113 profesores que realizaban las clases de música en el nivel de sexto básico. En Chile, no existe un catastro objetivo sobre el número total de profesores de música. Para efectos de esta investigación nos basamos en el último registro realizado por el Ministerio de Educación, en el año 2013. Se establece un total de 1.922 profesores de música a lo largo de todo el país.

La selección de los participantes se realizó en primer lugar gracias a la base de datos con que cuenta la agrupación de profesores de música Fladem Chile. En segundo lugar, vía redes sociales (foros de profesores de Chile, Facebook, Twitter). Se recibieron un total de 121 respuestas con la intención de participar, sin embargo 113 profesores cumplían con el perfil requerido para responder el cuestionario. El perfil requerido consistía en dar clases de música al nivel de sexto básico al menos desde el año 2011.

Los docentes que finalmente participaron fueron 101 profesores titulados de la carrera de pedagogía en educación musical, y 12 profesores titulados de pedagogía en enseñanza general básica. Además, entre los profesores de música titulados había quienes tenían segundas carreras tales como licenciatura en música (18 participantes) o interpretación instrumental o vocal (10 participantes). 
La elaboración del cuestionario buscaba obtener datos sobre la relación del docente con la música tradicional y su labor en el aula respecto a la utilización de la música tradicional como recurso de aprendizaje.

Tabla 2. Resumen de participantes de la encuesta

\begin{tabular}{|l|l|l|}
\hline Tipología profesional & Regiones donde trabajan & Tipo de institución en la que trabajan \\
\hline $\begin{array}{l}\text { 101 profesores música (titulados } \\
\text { de la carrera) }\end{array}$ & $\begin{array}{l}\text { Profesionales de 13 de las 15 } \\
\text { 12 profesores de enseñanza } \\
\text { general básica (titulados de la } \\
\text { carrera) }\end{array}$ & $\begin{array}{l}\text { Profesionales de establecimientos } \\
\text { municipales, subvencionados y } \\
\text { particulares. }\end{array}$ \\
& & \\
\hline
\end{tabular}

\section{Resultados}

A continuación, se presenta el análisis realizado, principalmente desde la visión del profesorado respecto a la utilización de la música tradicional en la escuela. Los docentes que participaron en esta encuesta fueron consultados sobre la valoración que ellos asignan a la enseñanza de las músicas tradicionales de diferentes lugares de Chile, dato de vital importancia para el diagnóstico que se buscaba en esta fase de la investigación. Para el desarrollo de este artículo, han sido consideradas sólo parte de las preguntas realizadas a los docentes participantes con el objetivo de conocer la utilización de las músicas tradicionales en la clase de educación musical.

Pregunta 15: Con el programa de estudios actual, ¿Qué cantidad de tiempo del año académico destinas al tratamiento de las músicas tradicionales de Chile u otros lugares de Latinoamérica?

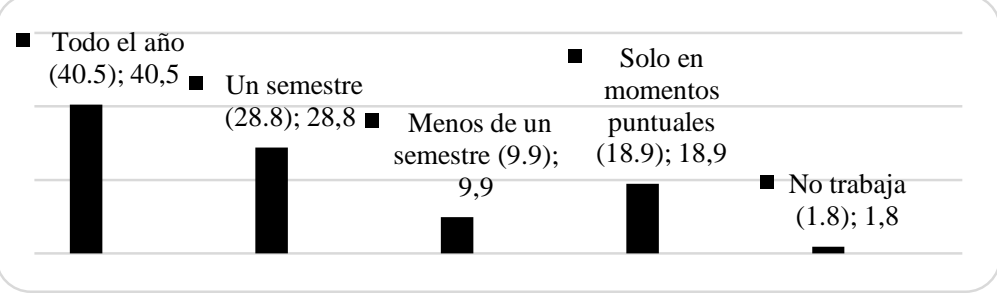

Figura 1. Tiempo destinado a la música tradicional

Se obtuvieron un total de 111 respuestas por parte de los docentes. Como se observa en la Figura 1, un 40.5\% (45 respuestas) afirma trabajar la música tradicional durante todo el año. El resultado llama la atención, ya que, con el actual programa de estudio para este nivel de enseñanza, la música tradicional no tiene un lugar claro y definido durante el transcurso de los

(c) HITA XIMENA VALVERDE OCARIZ Y PERE GODALL. THE CONTENT OF THIS ARTICLE IS THE SOLE RESPONSIBILITY OF THE AUTHORS. THE REVISTA ELECTRÓNICA DE LEEME AND UNIVERSITAT DE VALENCIA ARE NOT LIABLE FOR ANY LEGAL ACTIONS THAT MAY ARISE INVOLVING THE ARTICLE'S CONTENT. REVISTA ELECTRÓNICA DE LEEME -LISTA ELECTRÓNICA EUROPEA DE MÚSICA EN LA EDUCACIÓN-HTTP://MUSICA.REDIRIS.ES.ISSN: 1575-9563 EDITORES: UNIVERSIDAD DE VALENCIA Y JESÚS TEJADA GIMÉNEZ. VISIBILIDAD DE ESTA REVISTA: EBSCO, CINDOC (CESIC), CITEFACTOR, COPAC, DIALNET, DICE (CSIC), DOAJ, E-REVISTAS (CSIC), EBSCO PREMIER, ERIH+, GALE CENGAGE LEARNING, IN-RECS, IRESIE, LATINDEX, MIAR, OCLC WORLDCAT, RESH, REDIB, RILM CORE JOURNALS, SUDOC, ULRICHS, ESTA REVISTA ESTÁ PUBLICADA CON EL APOYO INSTITUCIONAL DE REDIRIS-CONSEJO SUPERIOR DE INVESTIGACIONES CIENTIFICAS Y ES DE ACCESO LIBRE. CREATIVE COMMONS LICENSE 4.0 BY 
aprendizajes. Por otro lado, un $18.9 \%$ (21 respuestas), afirma trabajarlo en momentos específicos del año, esto quiere decir según fundamentan algunas respuestas, que estas músicas son trabajadas especialmente en el denominado "mes de la patria". Esto ocurre en el mes de septiembre donde en colegios de todo Chile se celebra el comienzo del proceso de independencia nacional (1810), el cual es enarbolado con alegorías nacionalistas y donde en ese momento se hacen visibles músicas folclóricas y tradicionales de diferentes lugares de Chile.

Pregunta 18: Consideramos de importancia conocer las estrategias y recursos que los profesores utilizan en sus clases al momento de utilizar músicas tradicionales. En el cuestionario se incluyó la pregunta ¿Qué recursos utilizas en tus clases para abordar las músicas tradicionales? Los participantes debían jerarquizar en una lista de 9 tipos de recursos diferentes entre los cuales se contaban; la ejecución instrumental, canto colectivo y/o individual, invitación a cultores (expertos en la tradición sin estudios formales) al aula, danza y/o expresión corporal, salidas a terreno, investigaciones con los estudiantes, algún soporte multimedia, videos documentales, clases expositivas.

Para los participantes de la encuesta, el recurso más utilizado es la ejecución instrumental, situándose en el primer lugar. El canto colectivo y/o individual ocupa el segundo lugar en la jerarquía. Por el lado opuesto el recurso menos utilizado, corresponde a la participación de cultores en el aula donde ocupa siempre el noveno lugar en la jerarquía. Otro recurso muy poco utilizado es la danza y la expresión corporal situándose en el séptimo lugar. Estos datos refuerzan nuestro planteamiento respecto a la disparidad en la formación del profesorado donde el tratamiento de la música tradicional es actualmente escaso.

Pregunta 22 y 23: Otra de las preguntas que analizadas tiene relación con la importancia que el docente asigna en la clase al tratamiento de estas músicas: ¿Qué nivel de importancia otorgas a la música tradicional en tus clases?

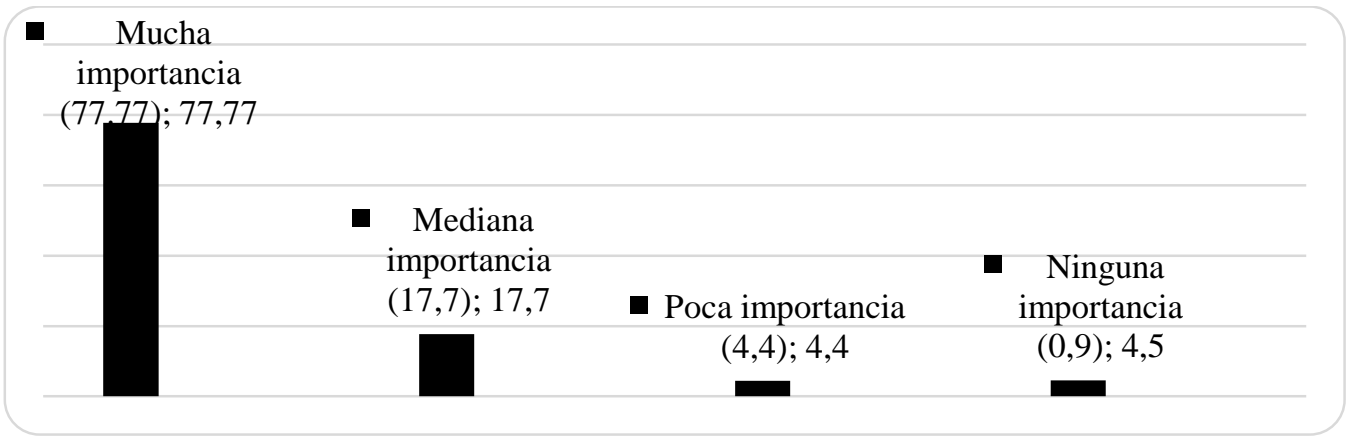

Figura 2. Importancia de la música tradicional en la clase

Se obtuvieron un total de 113 respuestas. En base a las respuestas obtenidas, es evidente que para un porcentaje importante de los participantes el tratamiento de estas músicas en el aula es muy importante $(77,77 \%)$. Más aún, cuando se invita a los participantes a fundamentar la razón de su respuesta nos encontramos con variadas opiniones entre las cuales destacamos las que

(c) HITA XIMENA VALVERDE OCARIZ Y PERE GODALL. THE CONTENT OF THIS ARTICLE IS THE SOLE RESPONSIBILITY OF THE AUTHORS. THE REVISTA ELECTRÓNICA DE LEEME AND UNIVERSITAT DE VALËNCIA ARE NOT LIABLE FOR ANY LEGAL ACTIONS THAT MAY ARISE INVOLVING THE ARTICLE'S CONTENT. REVISTA ELECTRÓNICA DE LEEME —LISTA ELECTRÓNICA EUROPEA DE MÚSICA EN LA EDUCACIÓN-HTTP://MUSICA.REDIRIS.ES.ISSN: 1575-9563 EDITORES: UNIVERSIDAD DE VALENCIA Y JESÚS TEJADA GIMÉNEZ. VISIBILIDAD DE ESTA REVISTA: EBSCO, CINDOC (CESIC), CITEFACTOR, COPAC, DIALNET, DICE (CSIC), DOAJ, E-REVISTAS (CSIC), EBSCO PREMIER, ERIH+, GALE CENGAGE LEARNING, IN-RECS, IRESIE, LATINDEX, MIAR, OCLC WORLDCAT, RESH, REDIB, RILM CORE JOURNALS, SUDOC, ULRICHS, ESTA REVISTA ESTÁ PUBLICADA CON EL APOYO INSTITUCIONAL DE REDIRIS-CONSEJO SUPERIOR DE INVESTIGACIONES CIENTIFICAS Y ES DE ACCESO LIBRE. CREATIVE COMMONS LICENSE 4.O BY 


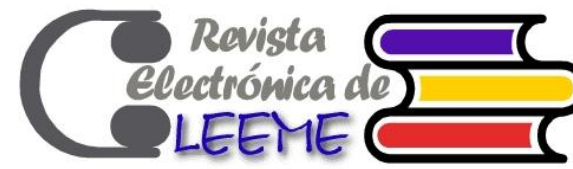

indican que a través de la enseñanza de estas músicas se buscan objetivos tales como el desarrollo de una identidad y pertenencia nacional:

"Es importante poder entregar a los alumnos un conocimiento sobre los distintos tipos de música tradicional del país, ya que deben tener un conocimiento, aunque sea mínimo del tema, ya que les entrega las herramientas necesarias para poder crear identidad, pertenencia, patrimonio, tolerancia e integración, la cual en estos tiempos es cada vez más necesaria”.

(Profesor de música, región de Coquimbo Chile)

Pregunta 28: Finalmente se analiza una pregunta abierta realizada en la encuesta, la cual pretendía conocer cuál es la postura que tiene el docente frente a la utilización de estas músicas como recursos de aprendizaje en el aula. La pregunta se planteó de la siguiente forma:

Opinión final: Entregamos este espacio para permitir al docente expresar alguna opinión personal en relación a la presencia de la música tradicional en el aula de $6^{\circ}$ básico, que no haya sido considerada en las preguntas anteriores. Para esta pregunta fue posible generar algunas categorías relacionadas con el tipo de respuestas otorgadas por los profesores participantes, las cuales surgieron en base al análisis del contenido de las respuestas obtenidas y se propusieron a partir de las similitudes y reiteración de estas. La Tabla 3 resume las tipologías de respuesta obtenida:

Tabla 3. Tipologías de docentes según respuestas

\begin{tabular}{|c|c|}
\hline Tipología del docente & Descripción \\
\hline Profesor nacionalista & $\begin{array}{l}\text { Busca desarrollar un sentimiento de chilenidad y patriotismo en la clase, a partir de la } \\
\text { utilización de la música tradicional. Propicia y motiva al estudiante a desarrollar una } \\
\text { identidad nacional. }\end{array}$ \\
\hline $\begin{array}{l}\text { Profesor transmisor y } \\
\text { conservador }\end{array}$ & $\begin{array}{l}\text { Siente que su rol está en la transmisión y la conservación de músicas tradicionales y la } \\
\text { clase de música es un espacio importante para este desarrollo. Se diferencia del profesor } \\
\text { nacionalista porque no persigue el desarrollo de un concepto de chilenidad, sino que } \\
\text { aboga por la conservación de las tradiciones sin importar el país. }\end{array}$ \\
\hline Profesor integrador & $\begin{array}{l}\text { Busca acercarse a los estudiantes inicialmente valorando las experiencias musicales que } \\
\text { traen desde fuera del contexto educativo formal, sin importar que tipo de música sea } \\
\text { esta. En una siguiente etapa este profesor acerca a los estudiantes a las músicas } \\
\text { tradicionales. Además, comprende que el valor de la música tradicional va más allá de } \\
\text { lo musical y considera como un aporte para el desarrollo humano. }\end{array}$ \\
\hline Profesor contracultural & $\begin{array}{l}\text { Defiende la importancia de la inclusión de la música tradicional para contrapesar toda } \\
\text { la cultura foránea a la que están expuestos los estudiantes fuera de la escuela. Este } \\
\text { profesor además tiene un compromiso y se siente una pieza fundamental como } \\
\text { transmisor de cultura a los estudiantes. }\end{array}$ \\
\hline Profesor pragmático & $\begin{array}{l}\text { No tiene un interés particular por desarrollar este tipo de música (tradicional), ya que su } \\
\text { interés mayor se encuentra en el desarrollo musical de los estudiantes, independiente } \\
\text { del repertorio que se utilice (sin embargo, no la descarta). }\end{array}$ \\
\hline
\end{tabular}

Por último, en relación a la pregunta anterior, llama la atención que, dentro de las respuestas

(c) HITA XIMENA VALVERDE OCARIZ Y PERE GODALL. THE CONTENT OF THIS ARTICLE IS THE SOLE RESPONSIBILITY OF THE AUTHORS. THE REVISTA ELECTRÓNICA DE LEEME AND UNIVERSITAT DE VALĖNCIA ARE NOT LABLE FOR ANY LEGAL ACTIONS THAT MAY ARISE INVOLVING THE ARTICLE'S CONTENT. REVISTA ELECTRÓNICA DE LEEME - ISTA ELECTRÓNICA EUROPEA DE MÚSICA EN LA EDUCACIÓN-HTTP://MUSICA.REDIRIS.ES.ISSN: 1575-9563 EDITORES: UNIVERSIDAD DE VALENCIA Y JESÚS TEJADA GIMÉNEZ. VISIBILIDAD DE ESTA REVISTA: EBSCO, CINDOC (CESIC), CITEFACTOR, COPAC, DIALNET, DICE (CSIC), DOAJ, E-REVISTAS (CSIC), EBSCO PREMIER, ERIH +, GALE CENGAGE LEARNING, IN-RECS, IRESIE, LATINDEX, MIAR, OCLC WORLDCAT, RESH, REDIB, RILM CORE JOURNALS, SUDOC, ULRICHS, ESTA REVISTA ESTÁ PUBLICADA CON EL APOYO INSTITUCIONAL DE REDIRIS-CONSEJO SUPERIOR DE INVESTIGACIONES CIENTIFICAS Y ES DE ACCESO LIBRE. CREATIVE COMMONS LICENSE 4.O BY 


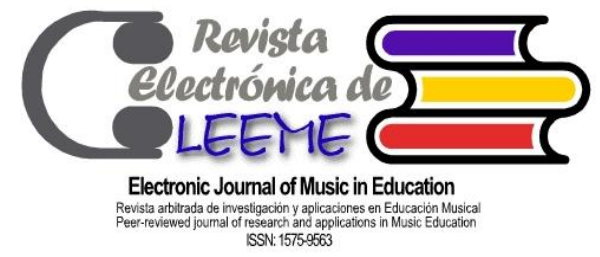

HITA XIMENA VALVERDE OCARIZ Y PERE GODALL

obtenidas, en al menos un tercio de los docentes existe una aparente confusión entre lo que se entiende por música tradicional y música de raíz folclórica. Estos docentes afirman que dentro de sus clases enseñan música de Víctor Jara o Violeta Parra, y a estas músicas las consideran como música tradicional. Aunque, por otro lado, es importante destacar que existe un interés por la enseñanza de estas músicas en el aula con el objetivo claro de desarrollar en los estudiantes un sentido de identidad cultural y pertenencia. Y aún más; otro grupo de respuestas contemplan la música tradicional como un medio para aprendizajes interdisciplinares y de recursos musicales que permiten a los estudiantes alcanzar mayores habilidades en esta área.

"La música latinoamericana y tradicional es parte de mis propias búsquedas. Corresponde a mi mundo significante. El enseñar sólo la música se vuelve una práctica inocua, en cambio el enseñar el sentido espiritual o más profundo del porque existen las músicas tradicionales otorga sentido y valor".

(Profesor de educación musical, región de Valparaíso Chile)

La respuesta anterior ejemplifica la responsabilidad que recae sobre el docente, como transmisor o no de estos aprendizajes. Cuando el docente tiene un nivel de compromiso como el antes expuesto, los estudiantes se verán beneficiados. El problema surge cuando el docente o bien no tiene el interés, o no cuenta con las herramientas necesarias para este propósito.

\section{Discusión}

Los datos y resultados aquí presentados han puesto de relieve elementos y evidencias que reafirman la importancia y significancia que la música tradicional puede llegar a tener en la escuela, tal como argumentan Small (1989), Casals (2009) y Campbell (2013). Por un lado, para enriquecer los conocimientos musicales de los estudiantes o, por el otro, y de vital importancia e interés educativo, para el acercamiento a los contextos socioculturales. Contextos donde estas músicas se dan y que permiten a los estudiantes ampliar los horizontes del conocimiento a partir de la disciplina de la música, pero con una visión integral; sin embargo, estos aprendizajes serán significativos siempre y cuando sean producto de un proceso de reflexión y contextualización.

Según lo propuesto por Abrahams (2008), Lines (2009) y Davis (2009), es importante comenzar a plantearnos como docentes algunas cuestiones fundamentales en cuanto al rol y las responsabilidades que tenemos dentro del aula y en las músicas que enseñamos a nuestros estudiantes. Finalmente, y no menos importante, existe el rol evidente que juega el Estado como la entidad que debe garantizar a sus ciudadanos una enseñanza de calidad. A continuación, se organiza la discusión en torno a estas tres cuestiones.

\section{La importancia del trabajo con músicas tradicionales en el aula}




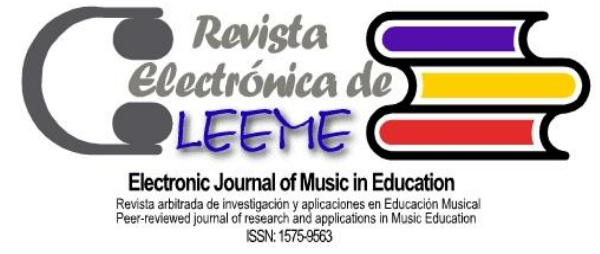

HITA XIMENA VALVERDE OCARIZ Y PERE GODALL (REVISTA ELECTRÓNICA DE LEEME) NÚMERO 41 LA PS. $16-34$ HTTPS://OJS.UV.ES/INDEX.PHP/LEEME/INDEX

De acuerdo con el análisis de los programas de estudio anteriores al año 2012, la utilización de músicas tradicionales en el aula de música no solamente aporta elementos para el trabajo musical, sino que se convierten en un elemento valioso para acercar a los estudiantes al conocimiento de la cultura. En este sentido, coincide con la opinión de Small (1989:87) quien afirma que la música puede constituirse como el indicador más sensible de la cultura debido a su estrecha vinculación con actitudes y supuestos inconscientes sobre los cuales construimos nuestra vida en el seno de una sociedad. La utilización de estas músicas en la escuela permite abordar diferentes tipos de aprendizajes, ya sean musicales o de carácter transversal los que contribuyen al desarrollo integral de los aprendizajes escolares. Sin embargo, es importante señalar que es necesario tener los recursos para poder desarrollar estos conocimientos. Éstos contribuirán a enriquecer el trabajo en el aula. Por ejemplo, la participación de un cultor en el aula para explicar desde su experiencia el rol que cumplen las músicas tradicionales en los lugares en que estas se desarrollan, puede contribuir de manera significativa al aprendizaje de los estudiantes. Por otro lado, consideramos la ejecución instrumental y vocal como el punto central dentro de la experiencia musical escolar. Sin embargo, somos conscientes de la importancia que tiene la danza en el desarrollo de estas músicas y vemos la poca inclusión que tiene dentro de la clase de música. Aún un paso más allá es proponer a incorporación de trabajos de campo o actividades en terreno, donde los estudiantes puedan participar de fiestas tradicionales que se realizan durante todo el año en Chile, incluso en lugares urbanos. De esta manera se incrementaría la experiencia de aprendizaje a partir de la experiencia etnográfica como una estrategia de aprendizaje.

\section{El rol del profesor frente al desafío de enseñar estas músicas}

Los datos recopilados y analizados nos permitieron identificar el punto donde se sitúa el docente, frente a su rol como mediador de aprendizajes en el aula. Abrahams (2008:307) afirma que cuando los docentes analizan, adaptan y manipulan el curriculum, abren la puerta a experiencias creativas que pueden ser liberadoras y transformadoras. Sin embargo, nuevamente reconocemos, que actualmente, debido a la libertad de enseñanza que no solo rige las escuelas chilenas sino también en las universidades, los profesores de educación musical deben enfrentarse en sus aulas a desafíos educacionales, y también musicales. En lo referido a las músicas tradicionales y al tratamiento de éstas en el aula, evidenciamos que en la mayoría de las universidades chilenas que imparten las carreras de pedagogía en música las asignaturas relacionadas con la música tradicional son escasas y, en algunos casos, simplemente no existen. De este modo, como lo hemos advertido durante el desarrollo de este artículo, la responsabilidad de ofrecer estos aprendizajes en el aula queda en manos del profesor de música o, en el caso que la clase no sea realizada por el profesor de música, esta responsabilidad recae en el profesor de educación general básica, quien cuenta con una menor preparación en la disciplina.

Creemos que es fundamental la creación de espacios de discusión, reflexión, reciclaje y/o adquisición de nuevos conocimientos por parte de los docentes en temáticas relacionadas con el

(c) HITA XIMENA VALVERDE OCARIZ Y PERE GODALL. THE CONTENT OF THIS ARTICLE IS THE SOLE RESPONSIBILITY OF THE AUTHORS. THE REVISTA ELECTRÓNICA DE LEEME AND UNIVERSITAT DE VALËNCIA ARE NOT LIABLE FOR ANY LEGAL ACTIONS THAT MAY ARISE INVOLVING THE ARTICLE'S CONTENT. REVISTA ELECTRÓNICA DE LEEME —LISTA ELECTRÓNICA EUROPEA DE MÚSICA EN LA EDUCACIÓN-HTTP://MUSICA.REDIRIS.ES.ISSN: 1575-9563 EDITORES: UNIVERSIDAD DE VALENCIA Y JESÚS TEJADA GIMÉNEZ. VISIBILIDAD DE ESTA REVISTA: EBSCO, CINDOC (CESIC), CITEFACTOR, COPAC, DIALNET, DICE (CSIC), DOAJ, E-REVISTAS (CSIC), EBSCO PREMIER, ERIH+, GALE CENGAGE LEARNING, IN-RECS, IRESIE, LATINDEX, MIAR, OCLC WORLDCAT, RESH, REDIB, RILM CORE JOURNALS, SUDOC, ULRICHS, ESTA REVISTA ESTÁ PUBLICADA CON EL APOYO INSTITUCIONAL DE REDIRIS-CONSEJO SUPERIOR DE INVESTIGACIONES CIENTIFICAS Y ES DE ACCESO LIBRE. CREATIVE COMMONS LICENSE 4.0 BY 


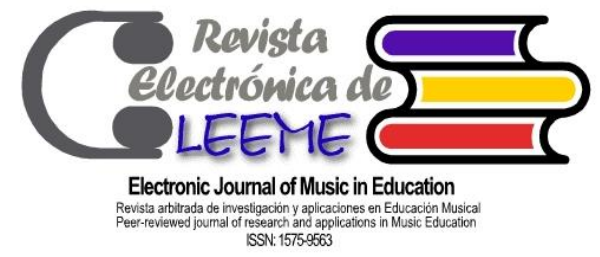

HITA XIMENA VALVERDE OCARIZ Y PERE GODALL (REVISTA ELECTRÓNICA DE LEEME) NÚMERO 41 , PP. $16-34$ HTTPS://OJS.UV.ES/INDEX.PHP/LEEME/INDEX

tratamiento de las músicas tradicionales en el aula. Este tipo de aprendizajes, indudablemente, nos llevan a un trabajo interdisciplinar puesto que los estudiantes no solamente aprenden sobre música, sino que además están acercándose a las costumbres, tradiciones e incluso las cosmovisiones que tienen los pueblos de los diferentes lugares de Chile. Este tipo de iniciativas educacionales se pueden convertir en un momento perfecto para trabajar desde perspectivas multidisciplinares y, además, donde sea posible, involucrar y hacer partícipe a la totalidad de la comunidad educativa.

Hemos podido observar a lo largo de esta investigación y, durante los años de trabajo realizado en el aula de música de escuelas, que muchas veces la utilización de estas músicas carece de un fundamento ideológico en pos del reconocimiento y valoración cultural tanto de los pueblos originarios de Chile como así también de la propia cultura popular tradicional. En vez de eso las escuelas muchas veces optan por muestras "folclóricas" las cuales no cuentan con una verdadera fundamentación teórica y se transforman en objetos decorativos dentro de actividades de carácter nacionalista y solo en momentos específicos del año.

\section{El rol del Estado como educador y transmisor de cultura en la escuela}

Por último, y como una de las interrogantes que plantea esta investigación, es importante conocer donde se sitúa el Estado a través del Ministerio de Educación. El objetivo de este estudio no se centró en la valoración de los programas de estudios, sin embargo, nos preguntamos por qué se optó por un programa de estudio tan abierto y flexible, y, por otro lado, por qué ya no están presentes de forma explícita, los aprendizajes relacionados con la cultura tradicional, las músicas y costumbres de los pueblos originarios y también con la cultura popular del país. Así, surge la pregunta sobre la visión que tiene el Ministerio de Educación para y con la educación musical ya que, además, desde el año 2012, las horas para la educación musical en segundo ciclo de enseñanza básica fueron reducidas a 45 minutos semanales.

Esto se contrapone a lo que sucede en primer ciclo de enseñanza básica $\left(1^{\circ}\right.$ a $4^{\circ}$ básico $)$ donde si existió un aumento de las horas de educación musical, pero las cuales, en su mayoría son realizadas por los profesores de educación general básica, quienes como dijimos antes no cuentan con la formación necesaria. Esto sumado a que los programas de estudio actuales no cuentan con lineamientos sobre qué tipo de educación musical busca el Estado para sus ciudadanos; por el contrario, el Estado advierte en varios momentos que los programas son siempre flexibles, adaptables y contextualizables. Sin embargo, lo que se evidencia con facilidad, es delegar en el docente la responsabilidad de entregar uno u otro tipo de aprendizajes, y lamentablemente, los docentes reconocen que no siempre cuentan con las estrategias adecuadas para tratar diferentes tipos de músicas en el aula.

Finalmente, señalamos la importancia de generar futuras investigaciones en esta área de la educación musical, pese a que en otros lugares del mundo como por ejemplo Argentina o 


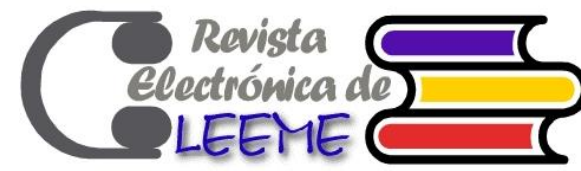

España, este es un tema de gran importancia. La realidad de la investigación en educación musical en Chile tiene por el momento otros intereses. Por otro lado, es importante asumir y reconocer las particularidades socioculturales que tiene el territorio chileno y como a partir de la música es posible llegar a puntos de consenso a nivel de construcción de identidades culturales colectivas en la escuela.

\section{Referencias}

Abrahams, F. (2008). "Musicando Paulo Freire: una pedagogía crítica para la educación musical". En P. McLaren y J. Kincheloe (Eds), Pedagogía crítica: de que hablamos, donde estamos (pp. 305-322). Barcelona: Graó.

Anderson, W. y Campbell, P. (1996). Multicultural Pespectives in music Education. Virginia: Menc.

Arévalo, A. (2009). Importancia del folklore musical como práctica educativa. Revista electrónica de Leeme, 23, 1-14.

Campbell, P. (2013). Etnomusicología y educación musical: punto de encuentro entre música, educación y cultura. Revista internacional de educación musical, 1, 42-52.

Casals, A. (2009). La cançó amb text improvisat. Disseny i experimentación d'una proposta interscipliplinària per a primària. Bellaterra: Universidad Autónoma de Barcelona.

Davis, R. (2009). "Educación musical e identidad cultural”. En D. K. Lines (Ed.), La educación musical para el nuevo milenio (pp. 71-90). Madrid: Morata.

Delgado, J. (2005). La aplicación de la música tradicional canaria en las aulas: un reto didáctico para el profesorado. Revista electrónica de Leeme, 15, 1-14.

Donoso, K. (2009). Por el arte-vida del pueblo: Debates en torno al folclor en Chile 1973-1990. Revista musical chilena, 212, 29-50.

Green, L. (2008). Informal learning and the school: a new classroom pedagogy. Hampshire: Ashgate.

Hargreaves, D. (1998). Música y desarrollo psicológico. Barcelona: Graó.

Inzunza, J., Assaél, J., y Scherping, G. (2011). Formación docente inicial y en servicio en Chile: tensiones de un modelo neoliberal. Revista mexicana de investigación educativa, 16 (48), 267292.

Lines, D. (2009). La improvisación y el trabajo cultural en la música y en la educación musical. En D. K. Lines (Ed.), La educación musical para el nuevo milenio (pp. 91-102). Madrid: Morata.

Martí, J. (2000). Más allá del arte. La música como generadora de realidades sociales. Sant Cugat: Deriva.

(c) HITA XIMENA VALVERDE OCARIZ Y PERE GODALL. THE CONTENT OF THIS ARTICLE IS THE SOLE RESPONSIBILITY OF THE AUTHORS. THE REVISTA ELECTRÓNICA DE LEEME AND UNIVERSITAT DE VALĖNCIA ARE NOT LIABLE FOR ANY LEGAL ACTIONS THAT MAY ARISE INVOLVING THE ARTICLE'S CONTENT. REVISTA ELECTRÓNICA DE LEEME -LISTA ELECTRÓNICA EUROPEA DE MÚSICA EN LA EDUCACIÓN-HTTP://MUSICA.REDIRIS.ES.ISSN: 1575-9563 EDITORES: UNIVERSIDAD DE VALENCIA Y JESÚS TEJADA GIMÉNEZ. VISIBILIDAD DE ESTA REVISTA: EBSCO, CINDOC (CESIC), CITEFACTOR, COPAC, DIALNET, DICE (CSIC), DOAJ, E-REVISTAS (CSIC), EBSCO PREMIER, ERIH+, GALE CENGAGE LEARNING, IN-RECS, IRESIE, LATINDEX, MIAR, OCLC WORLDCAT, RESH, REDIB, RILM CORE JOURNALS, SUDOC, ULRICHS, ESTA REVISTA
INVESTIGACIONES CIENTIFICAS Y ES DE ACCESO LIBRE. CREATIVE COMMONS LICENSE 4.0 BY 
Pino, O. (2015). El concepto de música en el curriculum escolar chileno 1810-2010. Tesis para optar al grado de Magister en Artes, mención Musicología. Santiago de Chile: Universidad de Chile.

Poblete, C. (2016). Profesores de música en Chile y sus repertorios: contextos de origen, formación universitaria y prácticas de enseñanza. Tesis para optar al grado de Doctor en Educación. Santiago de Chile: Pontificia Universidad Católica de Chile.

Salazar, N. (2016). Músicas tradicionales en espacios académicos: la rueda de gaita como experiencia de aprendizaje. Civilizar Ciencias Sociales y Humanas, 16 (31), 205-218.

Small, C. (1989). Música, sociedad y educación. Madrid: Alianza.

Valverde, X. (2013). Análisis de fortalezas y debilidades en relación al aporte de la Educación Artística en los subsectores de Matemáticas, Lenguaje y Ciencias de los docentes de primer ciclo básico del colegio Hispano Italiano de la ciudad de Iquique. Tesis de Magister en desarrollo curricular y proyectos educativos. Santiago de Chile: Universidad Andrés Bello.

(c) HITA XIMENA VALVERDE OCARIZ Y PERE GODALL. THE CONTENT OF THIS ARTICLE IS THE SOLE RESPONSIBILITY OF THE AUTHORS. THE REVISTA ELECTRÓNICA DE LEEME AND UNIVERSITAT DE VALĖNCIA ARE NOT LIABLE FOR ANY LEGAL ACTIONS THAT MAY ARISE INVOLVING THE ARTICLE'S CONTENT. REVISTA ELECTRÓNICA DE LEEME -LISTA ELECTRÓNICA EUROPEA DE MÚSICA EN LA EDUCACIÓN-HTTP://MUSICA.REDIRIS.ES.ISSN: 1575-9563 EDITORES: UNIVERSIDAD DE VALENCIA Y JESÚS TEJADA GIMÉNEZ. VISIBILIDAD DE ESTA REVISTA: EBSCO, CINDOC (CESIC), CITEFACTOR, COPAC, DIALNET, DICE (CSIC), DOAJ, E-REVISTAS (CSIC), EBSCO PREMIER, ERIH+, GALE CENGAGE LEARNING, IN-RECS, IRESIE, LATINDEX, INVESTIGACIONES CIENTIFICAS Y ES DE ACCESO LIBRE. CREATIVE COMMONS LICENSE 4.0 BY 
Anexos

\section{CUESTIONARIO N $^{\circ} 1$ "PRESENCIA Y TRATAMIENTO DE LA MÚSICA TRADICIONAL EN SEXTO AÑO BÁSICO"}

Estimad@s docentes: este cuestionario se propone en el marco de una investigación mayor. Este instrumento pretende identificar la presencia y tratamiento que se le otorga a la música tradicional en el aula de $6^{\circ}$ año básico.

*Para comprensión del término música tradicional utilizaremos aquellos que se presentan en los programas de estudio del año 1999 y 2012.

Podrán responderlo todas aquellas personas que hayan trabajado con el nivel de $6^{\circ}$ básico en los últimos dos años. Pedimos para ello tu colaboración, respondiendo las preguntas que se presentan a continuación. No implicará mucho tiempo de tu parte. Agradeceremos tu ayuda y garantizamos la confidencialidad de los datos.

\section{IDENTIFICACIÓN DEL PARTICIPANTE}

1. Nombre

2. Correo electrónico

3. Profesión u oficio

4. Universidad o instituto donde estudiaste

5. Años de experiencia como docente en aula

\begin{tabular}{|l|l|}
\hline & Es mi primer año de ejercicio profesional \\
\hline & Entre 1 y 5 años de experiencia profesional \\
\hline & Entre 6 y 10 años de experiencia profesional \\
\hline & Más de 10 años de experiencia profesional \\
\hline
\end{tabular}

6. Región o regiones donde trabajas actualmente

7. Nombre del establecimiento (s) donde trabajas

8. Tipo de establecimiento educacional

\begin{tabular}{|l|l|}
\hline & Municipal \\
\hline & Particular subvencionado \\
\hline & Particular \\
\hline
\end{tabular}

(c) HITA XIMENA VALVERDE OCARIZ Y PERE GODALL. THE CONTENT OF THIS ARTICLE IS THE SOLE RESPONSIBILITY OF THE AUTHORS. THE REVISTA ELECTRÓNICA DE LEEME AND UNIVERSITAT DE VALĖNCIA ARE NOT LIABLE FOR ANY LEGAL ACTIONS THAT MAY ARISE INVOLVING THE ARTICLE'S CONTENT. REVISTA ELECTRÓNICA DE LEEME -LISTA ELECTRÓNICA EUROPEA DE MÚSICA EN LA EDUCACIÓN-HTTP://MUSICA.REDIRIS.ES.ISSN: 1575-9563 EDITORES: UNIVERSIDAD DE VALENCIA Y JESÚS TEJADA GIMÉNEZ. VISIBILIDAD DE ESTA REVISTA: EBSCO, CINDOC (CESIC), CITEFACTOR, COPAC, DIALNET, DICE (CSIC), DOAJ, E-REVISTAS (CSIC), EBSCO PREMIER, ERIH+, GALE CENGAGE LEARNING, IN-RECS, IRESIE, LATIDEX, INVESTIGACIONES CIENTIFICAS Y ES DE ACCESO LIBRE. CREATIVE COMMONS LICENSE 4.0 BY 


\section{CONOCIMIENTO DE LOS PROGRAMAS DE ESTUDIO}

Esta sección aborda temas relacionados al conocimiento por parte del docente, respecto de "ambos" programas de Estudio para Educación Musical en $6^{\circ}$ año básico.

9. ¿Desarrollabas las unidades que proponía el Ministerio de Educación en el programa anterior de Educación musical para sexto básico?

\begin{tabular}{|l|l|l|l|l|l|l|l|}
\hline & Siempre & & A veces & & Ocasionalmente & & Nunca \\
\hline
\end{tabular}

10. ¿Utilizas el programa de Educación Musical para $6^{\circ}$ básico actual, para preparar tus clases y actividades de aula? Para esta pregunta por favor considera lo que efectivamente haces en la clase de música, no solamente aquello que escribes en la planificación.

\begin{tabular}{|l|l|l|l|l|l|l|l|}
\hline & Siempre & & A veces & & Ocasionalmente & & Nunca \\
\hline
\end{tabular}

11. Si tu respuesta anterior fue "nunca", podrías explicar brevemente ¿por qué no consideras su utilización?

\section{PRESENCIA DE MÚSICAS TRADICIONALES EN $6^{\circ}$ BÁSICO}

12. ¿Abordabas temáticas relacionadas con músicas tradicionales en $6^{\circ}$ básico a partir de la utilización de los programas de estudios anteriores? Pregunta referida a programa utilizado entre el año 1999 y 2011.

\begin{tabular}{|l|l|l|l|l|l|l|l|}
\hline & Siempre & & A veces & & Ocasionalmente & & Nunca \\
\hline
\end{tabular}

13. Si tu respuesta anterior fue "nunca", podrías explicarlo brevemente

14. ¿Qué cantidad de tiempo destinabas al trabajo de estos temas durante el año? Esta pregunta busca obtener una respuesta aproximada de lo realizado.

\begin{tabular}{|l|l|}
\hline & Lo trabajaba durante todo el año \\
\hline & Lo trabajaba durante todo un semestre \\
\hline & Lo trabajaba solo en una parte de un semestre \\
\hline & Lo trabajaba solo en momentos determinados durante el año \\
\hline & No lo trabajaba \\
\hline
\end{tabular}

15. Con el programa de estudios actual, ¿Qué cantidad de tiempo del año académico destinas al tratamiento de las músicas tradicionales de Chile u otros lugares de Latinoamérica?

\footnotetext{
\begin{tabular}{|l|l|}
\hline Lo abordo durante todo el año \\
\hline
\end{tabular}
} 


\begin{tabular}{|l|l|}
\hline & Lo abordo durante un semestre \\
\hline & Lo abordo por un periodo inferior a un semestre \\
\hline & Lo abordo solo en momentos específicos dentro del año \\
\hline & No lo abordo con sexto básico \\
\hline
\end{tabular}

\section{ESTRATEGIAS UTILIZADAS POR EL DOCENTE PARA EL TRATAMIENTO DE ESTAS MÚSICAS}

16. ¿Utilizas en tus clases músicas que se asocian a diferentes zonas geográficas de Chile o Latinoamérica?

\begin{tabular}{|l|l|}
\hline & $\mathrm{Si}$ \\
\hline & No \\
\hline
\end{tabular}

17. En relación a la pregunta anterior, ¿podrías explicar brevemente tu decisión?

18. ¿Qué recursos utilizas en tus clases para abordar las músicas tradicionales? Ordena de mayor a menor utilización. Marca solo una preferencia por fila.

\begin{tabular}{|l|l|l|l|l|l|l|l|l|l|}
\hline Estrategias/recursos & $1^{\circ}$ & $2^{\circ}$ & $3^{\circ}$ & $4^{\circ}$ & $5^{\circ}$ & $6^{\circ}$ & $7^{\circ}$ & $8^{\circ}$ & $9^{\circ}$ \\
\hline $\begin{array}{l}\text { Ejecución } \\
\text { instrumental }\end{array}$ & & & & & & & & & \\
\hline $\begin{array}{l}\text { Canto colectivo } \\
\text { y/o individual }\end{array}$ & & & & & & & & & \\
\hline $\begin{array}{l}\text { Invitación a } \\
\text { cultores al aula }\end{array}$ & & & & & & & & & \\
\hline $\begin{array}{l}\text { Danzas y/o } \\
\text { expresión } \\
\text { corporal }\end{array}$ & & & & & & & & & \\
\hline Salidas a terreno & & & & & & & & & \\
\hline $\begin{array}{l}\text { Investigaciones } \\
\text { con los } \\
\text { estudiantes }\end{array}$ & & & & & & & & & \\
\hline $\begin{array}{l}\text { Algún tipo de } \\
\text { soporte Tic }\end{array}$ & & & & & & & & & \\
\hline $\begin{array}{l}\text { Ver videos, } \\
\text { Documentales }\end{array}$ & & & & & & & & & \\
\hline $\begin{array}{l}\text { Clases } \\
\text { expositivas }\end{array}$ & & & & & & & & & \\
\hline
\end{tabular}

19. ¿Utilizas alguno de estos recursos para abordar las músicas tradicionales? Puedes marcar más de una preferencia si corresponde

\begin{tabular}{|l|l|}
\hline & $\begin{array}{l}\text { Realizo creaciones musicales a partir de elementos extraídos de estas músicas y los estudiantes las } \\
\text { interpretan vocal o instrumentalmente }\end{array}$ \\
\hline & Realizo arreglos y/o adaptaciones de músicas para que mis estudiantes las puedan tocar y/o cantar \\
\hline & $\begin{array}{l}\text { Los estudiantes realizan creaciones a partir de la utilización de elementos provenientes de las músicas } \\
\text { estudiadas }\end{array}$ \\
\hline
\end{tabular}

(C) HITA XIMENA VALVERDE OCARIZ Y PERE GODALL. THE CONTENT OF THIS ARTICLE IS THE SOLE RESPONSIBILITY OF THE AUTHORS. THE REVISTA ELECTRÓNICA DE LEEME AND UNIVERSITAT DE VALĖNCIA ARE NOT LIABLE FOR ANY LEGAL ACTIONS THAT MAY ARISE INVOLVING THE ARTICLE'S CONTENT. REVISTA ELECTRÓNICA DE LEEME -LISTA ELECTRÓNICA EUROPEA DE MÚSICA EN LA EDUCACIÓN-HTTP://MUSICA.REDIRIS.ES.ISSN: 1575-9563 EDITORES: UNIVERSIDAD DE VALENCIA Y JESÚS TEJADA GIMÉNEZ. VISIBILIDAD DE ESTA REVISTA: EBSCO, CINDOC (CESIC), CITEFACTOR, COPAC, DIALNET, DICE (CSIC), DOAJ, E-REVISTAS (CSIC), EBSCO PREMIR, L A , GILE CENGAGE LEARNING, IN-RECS, IRESIE, LATINDEX, INVESTIGACIONES CIENTIFICAS Y ES DE ACCESO LIBRE. CREATIVE COMMONS LICENSE 4.0 BY 
20. ¿Qué importancia les asignas al desarrollo del lenguaje musical (lecto escritura musical, teoría musical, entre otros) durante el desarrollo y tratamiento de las músicas tradicionales?

\begin{tabular}{|l|l|}
\hline & Lo considero de gran importancia \\
\hline & Lo considero de mediana importancia \\
\hline & Lo considero de menor importancia \\
\hline & No lo considero importante \\
\hline
\end{tabular}

21. ¿Con que frecuencia utilizas elementos de la danza y/o movimiento corporal para abordar estas unidades?

\begin{tabular}{|l|l|l|l|l|l|l|l|}
\hline & Siempre & & A veces & & Ocasionalmente & & Nunca \\
\hline
\end{tabular}

\section{INTERÉS DEL DOCENTE POR DIFUNDIR ESTAS MÚSICAS}

22. ¿Qué nivel de importancia otorgas a la música tradicional en tus clases?

\begin{tabular}{|l|l|l|l|l|l|}
\hline & 0 & 1 & 2 & 3 & \\
\hline Ninguna importancia & & & & & Mucha importancia \\
\hline
\end{tabular}

23. En relación a la pregunta anterior, ¿podrías explicar brevemente tu decisión?

24. ¿Has trabajando con alguna de estas músicas con $6^{\circ}$ básico? Esta pregunta se refiere específicamente al nivel de sexto básico. Puedes elegir más de una opción si es necesario

\begin{tabular}{|l|l|}
\hline & Música del pueblo Aymara \\
\hline & Música del pueblo Rapa Nui \\
\hline & Música del pueblo Mapuche \\
\hline & Música del pueblo Diaguita \\
\hline & Música del pueblo Atacameño \\
\hline & Otro: \\
\hline
\end{tabular}

25. ¿En qué medida estás de acuerdo con la siguiente afirmación?: "cuento con los elementos técnicos necesarios (formación) y didácticos (estrategias y recursos) necesarios para abordar la música tradicional en mis clases"

\begin{tabular}{|l|l|l|l|l|l|}
\hline & 0 & 1 & 2 & 3 & \\
\hline $\begin{array}{l}\text { Totalmente en } \\
\text { desacuerdo }\end{array}$ & & & & & Muy de acuerdo \\
\hline
\end{tabular}

(c) HITA XIMENA VALVERDE OCARIZ Y PERE GODALL. THE CONTENT OF THIS ARTICLE IS THE SOLE RESPONSIBILITY OF THE AUTHORS. THE REVISTA ELECTRÓNICA DE LEEME AND UNIVERSITAT DE VALĖNCIA ARE NOT LIABLE FOR ANY LEGAL ACTIONS THAT MAY ARISE INVOLVING THE ARTICLE'S CONTENT. REVISTA ELECTRÓNICA DE LEEME -LISTA ELECTRÓNICA EUROPEA DE MÚSICA EN LA EDUCACIÓN-HTTP://MUSICA.REDIRIS.ES.ISSN: 1575-9563 EDITORES: UNIVERSIDAD DE VALENCIA Y JESÚS TEJADA GIMÉNEZ. VISIBILIDAD DE ESTA REVISTA: EBSCO, CINDOC (CESIC), CITEFACTOR, COPAC, DIALNET, DICE (CSIC), DOAJ, E-REVISTAS (CSIC), EBSCO PREMIER, ERIH+, GALE CENGAGE LEARNING, IN-RECS, IRESIE, LATINDEX, MIAR, OCLC WORLDCAT, RESH, REDIB, RILM CORE JOURNALS, SUDOC, ULRICHS, ESTA REVISTA ESTÁ PUBLICADA CON EL APOYO INSTITUCIONAL DE REDIRIS-CONSEJO SUPERIOR DE INVESTIGACIONES CIENTIFICAS Y ES DE ACCESO LIBRE. CREATIVE COMMONS LICENSE 4.0 BY 
Electronic Journal of Music in Education

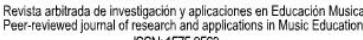

ISSN: 15759563

\section{PREGUNTAS DE CIERRE Y EVALUACIÓN DEL INSTRUMENTO}

26. En cuanto a comprender y responder este cuestionario, éste te ha resultado:

\begin{tabular}{|l|l|l|l|l|l|}
\hline & 0 & 1 & 2 & 3 & \\
\hline Muy dificil & & & & & Muy fácil \\
\hline
\end{tabular}

27. Responder este cuestionario te ha resultado:

\begin{tabular}{|l|l|l|l|l|l|}
\hline & 0 & 1 & 2 & 3 & \\
\hline Muy lento & & & & & Rápido \\
\hline
\end{tabular}

28. Opinión final: Entregamos este espacio para permitir al docente expresar alguna opinión personal en relación a la presencia de la música tradicional en el aula de $6^{\circ}$ básico, que no haya sido considerada en las preguntas anteriores.

\section{AGRADECEMOS PROFUNDAMENTE TU TIEMPO Y COLABORACIÓN EN ESTA INVESTIGACIÓN!!}

(c) HITA XIMENA VALVERDE OCARIZ Y PERE GODALL. THE CONTENT OF THIS ARTICLE IS THE SOLE RESPONSIBILITY OF THE AUTHORS. THE REVISTA ELECTRÓNICA DE LEEME AND UNIVERSITAT DE VALĖNCIA ARE NOT LIABLE FOR ANY LEGAL ACTIONS THAT MAY ARISE INVOLVING THE ARTICLE'S CONTENT. REVISTA ELECTRÓNICA DE LEEME -LISTA ELECTRÓNICA EUROPEA DE MÚSICA EN LA EDUCACIÓN-HTTP://MUSICA.REDIRIS.ES.ISSN: $1575-9563$ EDITORES: UNIVERSIDAD DE VALENCIA Y JESÚS TEJADA GIMÉNEZ. VISIBILIDAD DE ESTA REVISTA: EBSCO, CINDOC (CESIC), CITEFACTOR, COPAC, DIALNET, DICE (CSIC), DOAJ, E-REVISTAS (CSIC), EBSCO PREMIER, ERIH+, GALE CENGAGE LEARNING, IN-RECS, IRESIE, LATINDEX,
MIAR, OCLC WORLDCAT, RESH, REDIB, RILM CORE JOURNALS, SUDOC, ULRICHS, ESTA REVISTA ESTA PUBLICADA CON EL APOYO INSTITUCIONAL DE REDIRIS-CONSEJO SUPERIOR DE INVESTIGACIONES CIENTIFICAS Y ES DE ACCESO LIBRE. CREATIVE COMMONS LICENSE 4.0 BY 